Apidologie, 1979, 10 (1), 23-28.

\title{
L'INTÉRÊT DE L'ACÉTOLYSE EN MÉLISSOPALYNOLOGIE
}

\author{
Die Bedeutung der Acetolyse in der Melissopalynologie
}

\author{
Claude GADBIN \\ Laboratoire de Botanique historique et Palynologie, E.R.A. $n^{\circ} 404$ du C.N.R.S. \\ Faculté des Sciences et Techniques Saint-Jérôme, rue Henri-Poincaré, 13397 Marseille Cedex 4
}

\section{SUMMARY}

\section{INTEREST OF ACETOLYSIS IN MELISSOPALYNOLOGY}

The acetolysis method and its application to the pollen analysis of honey are described. The advantage of this technique is to permit a good and detailed observation of the exine structure of pollen grains, which is absolutely necessary in the case of geographical regions where the melliferous flora is not well known yet.

\section{RESUME}

La méthode de l'acétolyse est exposée ainsi que son application à la mélissopalynologie. Son intérêt est de permettre une observation fine et rigoureuse de la structure de la paroi pollinique, élément qui devient indispensable dans le cas des régions où la flore mellifère est mal connue.

Jusqu'en 1970, l'International Commission for Bee Botany (I.C.B.B.) de l'U.I.S.B. ne mentionne pas l'acétolyse du miel parmi les méthodes de mélissopalynologie. En 1965, MaUrizıo et LouveauX écrivent à propos des analyses polliniques en mélissopalynologie : «On ne procède à aucune fossilisation préalable, celle-ci n'étant pas utile en mélissopalynologie ".

Cette assertion est reprise par VoRwhol qui, en 1967, exclut l'acétolyse des méthodes d'analyse du miel " comme prenant trop de temps et provoquant la destruction d'éléments figurés accessoires tels que les algues, levures, morceaux d'insectes, inté- 
ressants pour l'étude du miel ». Les arguments de VoRwHOL demeurent valables pour l'étude des divers composants du miel, mais en mélissopalynologie plusieurs faits ont rendu nécessaire l'application des méthodes de traitement acétolytique mises au point par ERdTMAN (1936, 1943, 1952 et 1960) : d'une part, la recherche de l'origine florale de miels provenant de zones où la flore mellifère est inconnue; d'autre part, la rareté, pour ces régions, des pollens décrits - ces derniers étant, par ailleurs, toujours des pollens acétolysés.

Dès 1958, Vishnu-MitTre procède à des acétolyses sur des miels indiens. En 1972, les études de LiEux, en Louisiane, incluent l'acétolyse sans chlorination. Enfin, en 1976, SowuNmi, au Nigeria, affirme que l'acétolyse est indispensable à de telles études.

Après quelques essais d'analyse pollinique de miels originaires d'Afrique centrale, nous avons été amenés rapidement à réaliser des acétolyses de ces miels car, sans l'apport de cette technique, il était impossible d'identifier les pollens présents.

L'acétolyse, seule, permet, par la clarification des structures de la paroi pollinique qu'elle opère, une observation assez fine et notamment l'emploi correct de la L.O. analyse (REILle, 1970), support indispensable dans le cas de ces pollens exotiques mal connus.

Le projet de remaniement des " Méthodes de la mélisso-palynologie » nous a fourni l'occasion de préciser cette méthode.

\section{MATÉRIEL ET MÉTHODE}

Nous avons expérimenté notre méthode sur une dizaine de miels africains et sur cinq échantillons de miels français d'origine géographique différente. Dans tous les cas, le culot résultant du traitement a permis aisément une numération sur douze cents grains : nombre maximum de grains souhaité par l'I.C.B.B. et permettant une détermination des pourcentages d'espèces présentes (Louveaux, MaURizio, Vorwhol, 1970) très sûre.

$1{ }^{\circ}$ L'acétolyse selon Edtman (1943) est faite sur $10 \mathrm{~g}$ de miel, préalablement dissous dans $100 \mathrm{ml}$ d'eau tiède (pas plus de $40^{\circ}$ ) : centrifuger la solution obtenue. Décanter.

Le but de cette opération est d'éliminer aussi complètement que possible les sucres du miel qui noirciraient la préparation.

N.B. Toutes les centrifugations durent 10 minutes et se font à grande vitesse pour les petites centrifugeuses. Avec une centrifugeuse lourde, une vitesse de 2500 tours par minute pendant 5 minutes est suffisante.

La décantation est réalisée en versant doucement le liquide d'un mouvement régulier et continu, jusqu'à son élimination complète.

$2^{\circ}$ Sur le culot sont versés $10 \mathrm{ml}$ d'acide acétique pur. Après agitation et centrifugation l'acide est éliminé par décantation.

$3^{\circ}$ Sur ce culot sont alors ajoutés $2 \mathrm{ml}$ de "mélange acétolytique " : celui-ci confectionné peu avant son utilisation, dans une verrerie sèche, en versant goutte à goutte $0,2 \mathrm{ml}$ d'acide sulfurique pur dans $1,8 \mathrm{ml}$ d'anhydride acétique. 
L'ensemble est agité avec une baguette de verre sèche, puis placé pendant 5 à 10 minutes dans un bain-marie à $70^{\circ}$ (prendre garde de ne pas mettre en contact le mélange acétolytique et l'eau).

$4^{\circ}$ Sortir les tubes du bain-marie, bloquer l'acétolyse en ajoutant de l'acide acétique jusqu'à remplir le tube. Centrifuger et décanter.

$5^{\circ}$ Après centrifugation et décantation, le culot est rincé à l'eau distillée, agité, centrifugé à nouveau.

$6^{\circ}$ Le montage est réalisé dans la glycérine pure, milieu qui, s'il présente des inconvénients pour la conservation des pollens à long terme, a le grand avantage de permettre la rotation des pollens sous le microscope et leur étude selon plusieurs axes d'observation. On peut lui préférer le montage classique en mélisso-palynologie dans la gélatine glycérinée qui assure une meilleure conservation de la lame. Pour le matériel monté dans les pays tropicaux, ERDTMAN (1952) recommande le montage en glycérine pure.

\section{DISCUSSION}

Après avoir choisi d'acétolyser les pollens pour les raisons précédemment exposées, il a été effectué des comptages comparés sur deux miels, l'un traité selon les méthodes classiques de la mélissopalynologie et l'autre acétolysé, afin de vérifier que l'acétolyse n'entraîne pas la disparition sélective de certains types de pollen.

$1^{\circ}$ Ces comptages ont effectivement montré que le même nombre de types polliniques peuvent être distingués.

Lieu d'origine du miel soumis au test : Mailao (République du Tchad)

Date de récolte : Janvier 1975

Nombre de types polliniques distingués : miel acétolysé : 15 ; miel « frais " : 12 (comptage sur 500 pollens).

Il est cependant évident que ce test ne permet pas d'exclure l'hypothèse d'une substitution entre certains types polliniques mis en évidence par l'acétolyse et d'autres types polliniques existant dans le miel traité par les méthodes classiques mais non identifiés.

TABL. 1. - Fréquence relative des 5 taxons dont l'identification est aussi sûre sur le pollen frais que sur le pollen acétolysé.

TAB. 1. - Relative Häufigkeit von 5 Pollentypen, deren Bestimmung an frischen Pollenkörnern ebenso sicher ist wie an acetolysierten Körnern.

\begin{tabular}{|c|c|c|}
\hline Taxons & $\begin{array}{c}\text { Miel acétolysé } \\
\text { Acetolysierter Honig }\end{array}$ & $\begin{array}{c}\text { Miel frais } \\
\text { Frischer Honig }\end{array}$ \\
\hline 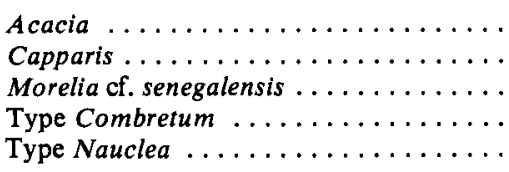 & $\begin{array}{r}28 \% \\
63 \% \\
1,47 \% \\
1,69 \% \\
0,25 \%\end{array}$ & $\begin{array}{cc}31 & \% \\
63 & \% \\
1,9 \% \\
1 & \% \\
0,25 \%\end{array}$ \\
\hline
\end{tabular}



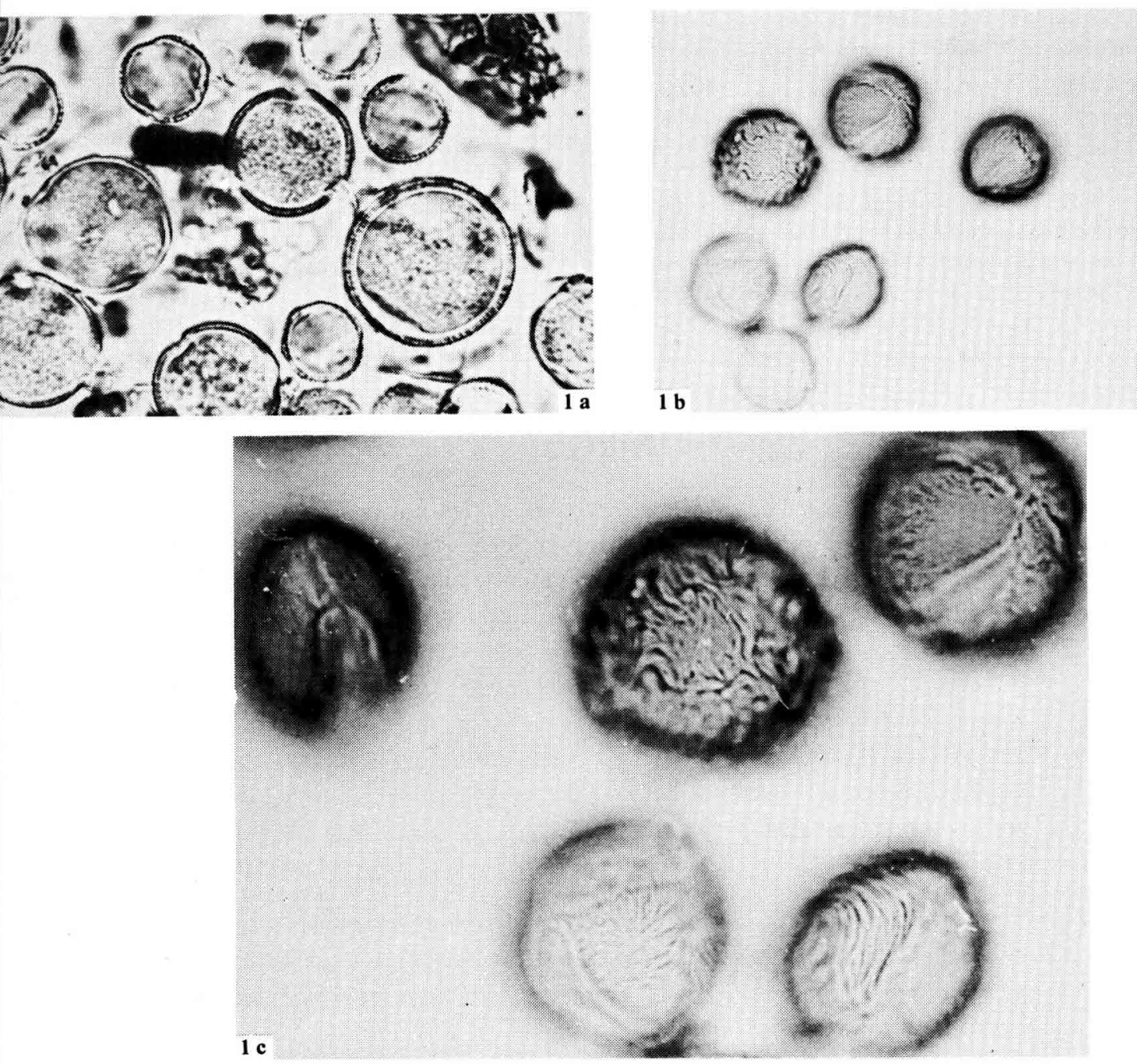

Fig. 1. - Miels originaires

du Tchad méridional.

FIG. 1a. - Miel traité selon la méthode mélissopalynologique classique. (X 400).

Fig. 1b. - Miel acétolysé (X 400).

FIG. 1c. - Miel acétolysé (X 1000).

Aвв. 1. - Honige aus dem südlichen Tschad.

Aв8. 1a. - Honigsediment behandelt nach der in der Honigpollenanalyse klassischen Methode (X 400).

AвB. 1b. - Acetolysiertes Honigsediment (X 400).

ABB. 1c. - Acetolysiertes Honigsediment (X 1000$)$. 
$2^{\circ}$ Un comptage qualitatif conduit à conclure à une représentation relative semblable des taxons dans les deux miels :

Lieu d'origine du miel soumis au test : Ndjamena (République du Tchad) Date de récolte : 27 février 1976

Nombre de types polliniques distingués : miel acétolysé : 13; miel frais : 11 (comptage sur 500 pollens).

\section{CONCLUSION}

Cette préparation, plus longue que le montage direct après rinçage préconisé par l'I.C.B.B. jusqu'en 1972, présente l'avantage incontestable de permettre une analyse pollinique s'appuyant sur une observation fine de la paroi pollinique, observation essentiellement basée sur la L.O. analyse, beaucoup plus rigoureuse et permettant, seule, en fonction des références existant dans la littérature, pour les pollens exotiques une identification spécifique. Elle a l'inconvénient d'éliminer certains éléments figurés du miel autres que les pollens dont l'observation sera toujours possible sur du miel traité selon la méthode classique.

Reçu pour publication en octobre 1978.

Eingegangen im Oktober 1978.

\section{ZUSAMMENFASSUNG}

Die Methode der Acetolyse (Säurebehandlung der Pollenkörner) und ihre Anwendung in der Pollenanalyse von Honigen werden beschrieben. Der Vorteil dieser Technik liegt darin, dass die Struktur der Exine (Schale) der Pollenkörner bis in alle Details sichtbar gemacht wird. Das ist in all jenen Gebieten unbedingt erforderlich, wo die Honigflora noch nicht ausreichend bekannt ist. Bei der Untersuchung von Honigen aus der Republik Tschad (Zentralafrika) konnten unter den acetolysierten Pollenkörnern mehr verschiedene Typen unterschieden werden als unter den nach der üblichen Methode behandelten.

Die vorherrschenden Pollentypen stammten von Capparis (63\%) und Acacia (28\%).

\section{BIBLIOGRAPHIE SOMMAIRE}

ERDTMAN G. 1935. - Investigation of honey pollen. Svensk Botanisk Tidskrift, 29 (1), 79-80.

Erdtman G., 1936. - Neue pollenanalytische Untersuchungsmethoden. Ber. Geobot. Forsch. Inst. Rubel, 1935.

ERdTMAN G., 1943. - An introduction to pollen analysis. Verdoorn, new ser. Pl. Sci. Books. 12. Waltham, Mass. 
Erdtman G., 1952. - Pollen morphology and plant taxonomy. Stockholm, Almquist et Wiksell. 539 p.

Erdtman G., 1960. - The acetolysis method. A revised description. Svensk Botanisk Tidskrift, 54 (4), $561-564$.

Lieux M. H., 1972. - A melissopalynological study of 54 Louisiana honeys. Rev. Paleobot. Palynol., 13 (3), 95-124.

LieUX M. H., 1975. - Dominant pollen types recorded from commercial Louisiana honeys.Econ. Bot., 29 (1), 87-96.

Louveaux J., Maurizio A., Vorwohl G., 1970. - Les méthodes de la mélissopalynologie. Apidologie, 1 (2), 193-209.

Maurizio A., Louveaux J., 1963. - Méthodes d'analyse pollinique des miels. Ann. A beille, 6 (1), 75-76.

REILlE M.; 1970. - Étude pollenanalytique de tourbières du Maroc. Recherches préliminaires et premiers résultats. Thèse spécialité. Marseille.

Sowunmi M. A., 1976. - The potential value of honey in paleopalynology and archeology. Rev. Paleobot. Palynol., 21 (2) 171-185.

VIEIrEz E., 1950. - Palynological observations on some spanish honeys. Bull. Torrey Botanical Club, 77 (6), 495-502.

VIeItez E., 1951. - El polen de las mieles de Galicia. Annales de Edafologia Vegetal, X (1), 79-100. Vishnu-Mittre, 1958. - Pollen content of some indian honey. J. Sci. Industr. Res. India, $17 \mathrm{c}(7), 123$ 124.

Vorwohl G., 1967. - The microscopic analysis of honey, a comparison of its methods with those of the other branches of palynology.Rev. Paleobot. Palynol., 3 (1-4), 287-290. 